\title{
Experimental Demonstration of TM Lateral Leakage in a Standard SOI Photonics Platform
}

\author{
Anthony P. Hope, ${ }^{1}$ Thach G. Nguyen, ${ }^{1}$ Wim Bogaerts, ${ }^{2 \dagger}$ and Arnan Mitchell ${ }^{1}$ \\ ${ }^{1}$ ARC Centre of Excellence for Ultrahigh bandwidth Devices for Optical systems (CUDOS) \\ and School of Electrical and Computer Engineering, RMIT University, Melbourne, Australia \\ ${ }^{2}$ Photonics Research Group, Ghent University-IMEC, department of Information Technology, \\ Center of Nano-and Biophotonics. 9000 Gent, Belgium, \\ ${ }^{\dagger}$ now also with: Luceda Photonics, Dendermonde, Belgium
}

\begin{abstract}
We provide an experimental demonstration of the width dependent losses of the fundamental TM guided mode in fabricated silicon-on-insulator shallow etched ridge waveguides.
\end{abstract}

\section{INTRODUCTION}

Shallowly etched ridge silicon-on-insulator (SOI) waveguides operating in TM polarisation exhibit lateral leakage effects [1] in which the power of the guided TM mode couples laterally into cladding TE slab modes propagating at a particular angle to the waveguide axis [1], [2]. Instead of viewing this lateral leakage as loss, it can be engineered for new photonic device topologies [3]. Previous demonstrations of lateral leakage waveguides rely on exotic and potentially non-CMOS compatible fabrication processes [1].

In this paper, we directly observe the lateral leakage phenomenon in fabricated shallow etched SOI waveguides using standard silicon photonic processing and demonstrate that the magnitude of the leakage depends on the waveguide geometry, strongly agreeing with simulations.

\section{LATERAL LEAKAGE IN SILICON RIDGE WAVEGUIDES}

High-contrast rib waveguides exhibit a peculiar loss behaviour for the TM polarisation, with a strong dependence on waveguide width $(\mathrm{w})$ [1]. Within the rib waveguide the TMlike mode has in-plane E-field components at the sidewalls,

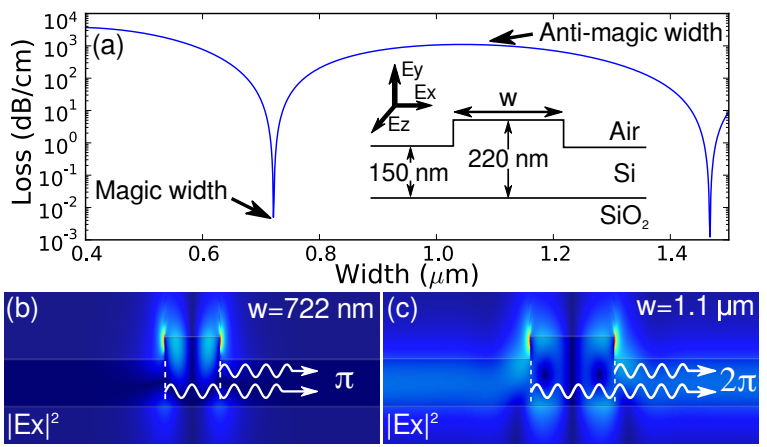

Fig. 1. (a) The simulated waveguide propagation loss of the TM mode as a function of the waveguide width (w) at a wavelength of $1550 \mathrm{~nm}$ inset: waveguide cross-section, (b) the lateral electric field of a 'magic-width' waveguide, and (c) of a strongly radiating waveguide. which gives rise to a non-zero overlap with the TE modes in the cladding. Also, as the TM has a lower effective refractive index than that of the TE slab modes in the cladding, there can be phase matching, appearing as radiative leakage of the TM waveguide mode [2]. This TM/TE coupling occurs at both sidewalls of the rib, and the two contributions will interfere. This can be either constructive (strong radiative leakage) or destructive (cancelling any radiative leakage), depending on the width of the rib. This width dependence is demonstrated in Fig. 1(a), and we can identify so-called 'magic' widths (no leakage, as shown in Fig. 1(b)) or 'antimagic' widths (Fig. 1(c)). The mode profiles were generated using RMITs Eigenmode Modelling Environment based on the film mode matching (FMM) method [2]. The waveguide TM mode radiates at specific angles in the slab corresponding to a matching of the k-vector along the axis of the waveguide. This depends strongly on the relative thicknesses of the waveguide core and the etched side cladding. Previous demonstrations of this phenomenon have relied on very shallow patterning (15 $\mathrm{nm}$ ) of the rib waveguides, using a local oxidation process [1]. In this paper, we demonstrate this effect in a standard silicon photonics platform and provide a quantitative characterisation.

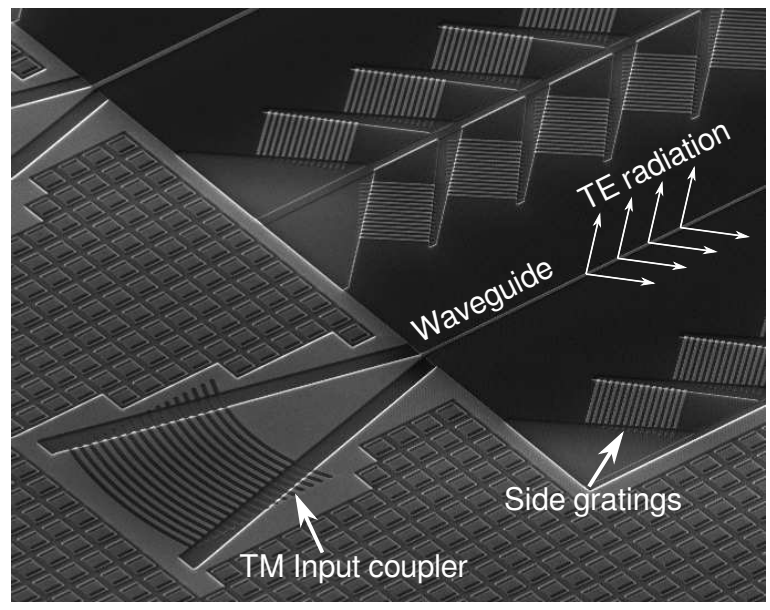

Fig. 2. SEM imaging of the fabricated lateral leakage SOI waveguide devices. 


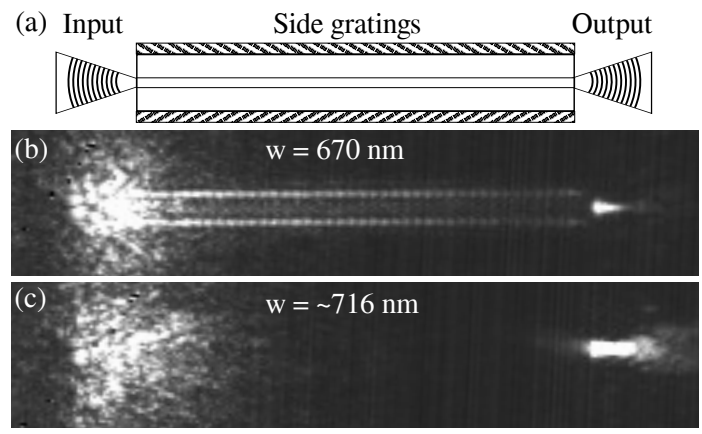

Fig. 3. Overhead infra-red imaging of $0.5 \mathrm{~mm}$ long waveguides; (a) Schematic of the observable area, (b) $w=670 \mathrm{~nm},(\mathrm{c}) \mathrm{w}=716 \mathrm{~nm}$.

\section{DESIGN AND FABRICATION OF SOI LATERAL LEAKAGE WAVEGUIDES}

We design a series of $70 \mathrm{~nm}$ shallow etched air-clad SOI waveguides on a $220 \mathrm{~nm}$ silicon slab with varying widths and lengths for characterisation. Based on the simulation results, the magic width for this geometry at a wavelength of $1550 \mathrm{~nm}$ is $722 \mathrm{~nm}$. The devices are designed using the parametric design framework IPKISS [4]. Fabrication is performed through ePIXfab using IMEC's passive silicon photonics platform [5]. The TM mode is accessed by input and output focussed grating couplers. To monitor the radiated light we include an array of 1-D grating couplers at the edge of the shallow etched slab. These couplers are designed to diffract the leaking TE light coming in laterally at approximately $45^{\circ}$ with the waveguide axis as mentioned earlier, and the out-of-plane diffraction direction is chosen to match that of the output coupler. Using these gratings, we can observe the leaking light with an infrared camera, or measure the intensity using a scanning fibre. Fig. 2 shows the SEM image of the fabricated devices.

\section{OBSERVATION OF LATERAL LEAKAGE BEHAVIOUR}

We obtain an initial view of the leakage behaviour using a vertically coupled fibre setup to input and an overhead infrared camera to observe any excitation of the gratings located at the slab boundaries and output port (Fig. 3(a)). The 670 $\mathrm{nm}$ width waveguides show strong excitation of these side TE gratings and a weakened output transmission (Fig. 3(b)) as compared to the $720 \mathrm{~nm}$ width waveguides which show no interaction with these side gratings and are very close to our expected magic width (Fig. 3(c)). The waveguides designed at $720 \mathrm{~nm}$ were measured with an SEM to be closer to $716 \mathrm{~nm}$.

Fig. 4 shows the measured power transmission of the fabricated waveguides at different widths and lengths. The waveguide widths presented in Fig. 4 are the widths measured from SEM imaging. Transmission measurements of the fabricated waveguides show clear evidence of a very low loss peak in the waveguide widths at $716 \mathrm{~nm}$ (Fig. 4(a)) which is in excellent agreement with the predicted 'magic' value shown in Fig. 1(a). As the length of the waveguides are increased to $1 \mathrm{~mm}$ the losses of the other waveguides increases dramatically away from this resonant width (Fig. 4(b)).
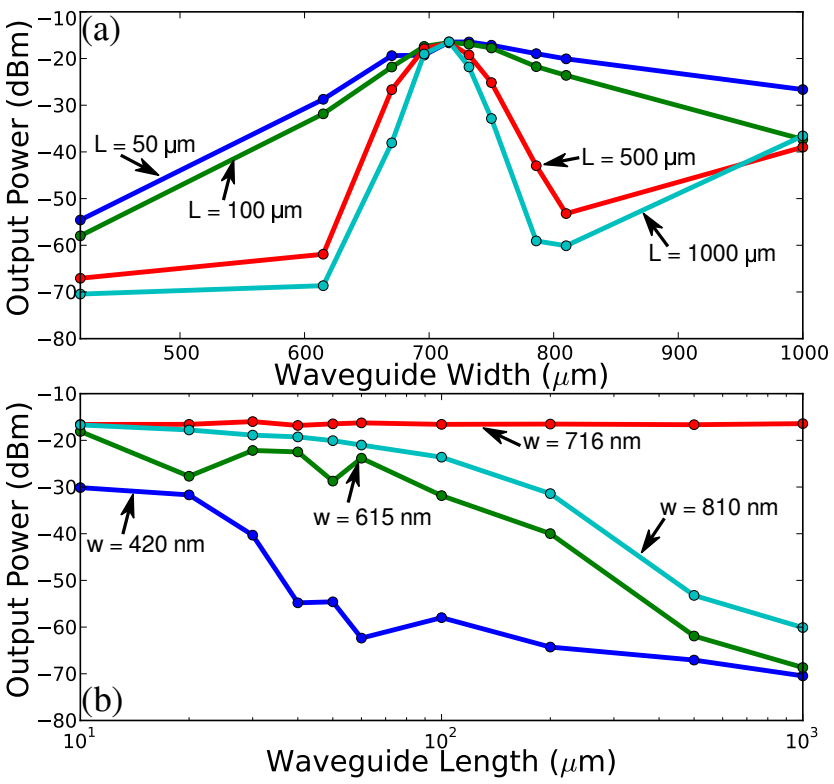

Fig. 4. Analysing the transmission of the waveguides with the wavelength fixed at $1550 \mathrm{~nm}$; (a) Varying the width of the waveguides at selected waveguide lengths, (b) transmission response of selected waveguide widths as the length is increased.

\section{CONClusion}

We have observed the width dependent lateral leakage present in shallow etched waveguides using a standard SOI photonics platform. The addition of angled gratings at the slab boundaries allows for the direct imaging of this interaction. Our fabricated devices show low loss propagation at $720 \mathrm{~nm}$ with the loss dramatically increasing away from this resonance, in close agreement with predictions. These results demonstrate that a standard silicon photonic platform can achieve controllable coupling between guided and radiative modes, which can potentially provide a routing pathway between two spatially separated waveguides, no longer relying on evanescent interactions [3].

\section{ACKNOWLEDGEMENT}

A.P.H, T.G.N and A.M. acknowledge the support of the Australian Research Council (ARC) Centre of Excellence Funding (CE110001018), A.P.H. acknowledges Robert and Josephine Shanks Scholarship, T.G.N. acknowledges support from the ARC APD fellowship (DP1096153).

\section{REFERENCES}

[1] M. Webster, R. Pafchek, A. Mitchell, et. al., IEEE Photon. Technol. Lett. 19(6), 429-431 (2007)

[2] T. Nguyen, R. Tummidi, T. Koch, et. al., Photon. Technol. Lett., IEEE 21, 486-488 (2009)

[3] A. Hope, T. Nguyen, A. Greentree, et. al., Opt. Express 21, 22705-22716 (2013).

[4] M. Fiers, E. Lambert, S. Pathak, et. al, J. of Comput. Sci., 4, 313-324 (2013).

[5] W. Bogaerts, S. Selvaraja, P. Dumon, et. al., J. Sel. Top. Quantum Electron. 16(1), 33-44 (2010) 